\title{
ENTRETIEN AVEC FRANÇOIS OST \\ DROIT ET LITTÉRATURE : LES DEUX FACES DU MIROIR
}

Par Dieter AXT ${ }^{1}$

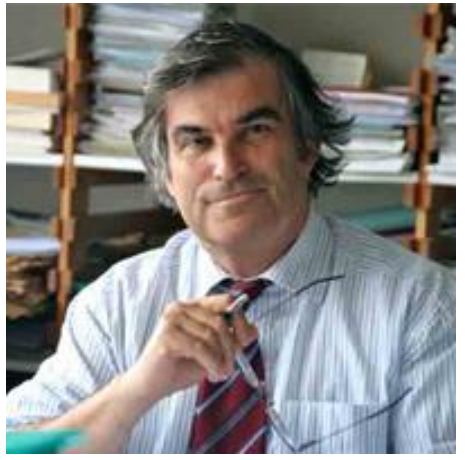

La SEÇÃo ENTREvistas a été conçue dans de but de créer un espace d'interlocution avec des chercheurs reconnus dans le domaine des études en Droit et Littérature, afin de promouvoir l'échange permanent d'idées et l'interaction de points de vue en rapprochant les chercheurs et les lecteurs.

Dans la présente édition d'Anamorphosis, nous avons le plaisir d'interviewer François Ost, l'un des pionniers et des principaux chercheurs de l'actualité en droit et littérature.

Né à Bruxelles en 1952, François Ost a une carrière solide et reconnue de juriste et de philosophe du droit. En 2001, il a reçu de l'Université de Nantes (France) le titre de Docteur honoris causa. En 2004, il a été élu membre de l'Académie royale des sciences, des lettres et des beaux-arts de Belgique. En 2006, son ouvrage Raconter la loi a obtenu le Prix quinquennal de l'essai de la Communauté française de Belgique. En 2010, il a reçu le Grand prix de la Fondation Prince Louis de Polignac pour l'ensemble de son œuvre.

Outre ses recherches au sein de l'Académie, François Ost est l'auteur de trois pièces de théâtre : Antigone voilée (Bruxelles, Larcier, 2004), La nuit la plus longue; Sade et Portalis au pied de l'échafaud (Bruxelles, Anthémis, 2008) et Camille (Carnières/Morlanwelz, Lansman, 2011).

1 Étudiant de master en droit public à l'Universidade do Vale do Rio dos Sinos (UNISINOS). Licencié en droit à l'Universidade Federal do Rio Grande do Sul (UFRGS). Scénariste de l'émission télévisée Direito \& Literatura (TV Justiça). Membre de la Rede Brasileira Direito e Literatura (RDL). Assistant d'édition d'Anamorphosis - Revista Internacional de Direito e Literatura. Écrivain et éditeur de la maison d'édition Le Chien. Porto Alegre, RS, Brasil. CV Lattes: http://lattes.cnpq.br/1582390811392545. E-mail: dieter@rdl.org.br 
Vice-recteur et professeur titulaire à l'Université Saint-Louis (Belgique), il est professeur titulaire à l'Université de Genève (Suisse) et à l'Université Catholique de Bruxelles (Belgique). Il a été professeur invité dans les universités de Louvain, de Buenos Aires, de São Paulo, de Macerata, de Paris-Nanterre et, de même, au Collège Belgique (Bruxelles), au Collège International de Philosophie (Paris), à l’École Nationale de la Magistrature (Bordeaux) et à l'Institute Universitaire Européen (Florence).

François Ost est, entre autres, co-directeur de l'Académie européenne de Théorie du Droit (Bruxelles), co-président de l'Association européenne pour l'enseignement de la Théorie du Droit (Bruxelles), président de la Fondation pour les Générations futures (Namur), membre-fondateur de l'Institut d'études sur la justice (Bruxelles).

Quelques-uns des ouvrages de son importante production bibliographique sont Le temps du droit (1999), Raconter la loi; Aux sources de limaginaire juridique (2004), Sade et la loi (2005), Dire le droit, faire justice (2007), La nature hors la loi. L'écologie à l'épreuve du droit (1995), Traduire; Défense et illustration du multilinguisme (2009), et Furetière, La démocratisation de la langue (2008). Certains de ses ouvrages ont été traduits en portugais : A natureza à margem da lei (Piaget, Lisboa), O tempo do direito (EDUSC, Bauru) e Contar a lei; as fontes do imaginário jurídico (UNISINOS, São Leopoldo).

Dieter Axt - Vous êtes l'un des pionniers et l'un des principaux représentants en ce qui concerne l'étude des relations entre le droit et la littérature. Qu'est-ce qui vous a motivé à débuter des études dans ce domaine et de quelle façon le droit et la littérature peuvent-ils converger?

Je faisais des cours, depuis longtemps, dans le domaine de la philosophie du droit. Je me suis aperçu que, bien souvent, j’introduisais l'exposé d'une question par un récit littéraire. Je pense par exemple à la question de la résistance à l'oppression et de la désobéissance civile que je mettais en rapport avec la tragédie d'Antigone de Sophocle. Mais les exemples sont nombreux: Faust et le contrat, Robinson Crusoë et la propriété privée, Richard II de Shakespeare et la doctrine « des deux corps du roi », Dickens et les juges iniques... 
A un certain moment je me suis persuadé que ces rapprochements ne résultaient pas du hasard, et je me suis mis à considérer sérieusement cette production artistique et littéraire. L'enjeu, au moment d'opérer ce basculement, est de ne pas se contenter de considérer la littérature comme un simple réservoir d'exemples en vue d'illustrer telle ou telle thèse de philosophie du droit. Il fallait prendre ces textes au sérieux, pour euxmêmes et dans leur intertextualité littéraire; ne pas seulement leur adresser des questions de juristes, mais se laisser aussi décentrer et interpeller par leur imaginaire propre. C'est une condition d'entrée indispensable dans la pratique interdisciplinaire, d'autant plus nécessaire qu'on rapproche ici, plutôt que deux sciences (science du droit et science littéraire), deux imaginaires et deux pratiques (pratique juridique et pratique de l'écriture littéraire ou théâtrale).

Droit et littérature convergent de multiples façons. Je me contente, dans cette première question, d'évoquer deux pistes de réponse. D'une part, le fait, avéré, que, dans leur formation et leur carrière, hommes de lettres et hommes de lois ne font souvent qu'un : singulièrement nombreux sont les auteurs ayant suivi une formation juridique (Balzac, Flaubert, Dickens,...), et pas mal d'entre eux ont même travaillé leur vie durant comme juriste (Kafka). L'autre piste consiste à souligner combien le raisonnement juridique, et particulièrement le raisonnement judiciaire, est pétri de narrativité : je pense au récit des faits en justice, mais aussi au récit des «précédents » (on songe à Dworkin et à ses juges, «conteurs moraux de la nation », rendant la justice sur le modèle de l'écriture « d'un roman à la chaîne »), je pense aussi au récit des connotations " encyclopédiques » qui s'attachent aux mots de la loi (cf. U. Eco insistant sur l'importance d'une interprétation basée sur cette histoire « encyclopédique », par opposition à une simple définition analytique «lexicale» de ces termes), je pense enfin au récit pragmatique des péripéties du procès lui-même et des divers avatars de sa procédure. C'est de part en part que la jurisdictio est pénétrée par le récit, qui est reconstruction imaginaire de son contexte et production narrative de son sens. 
Dieter Axt - No livro Contar a lei - as fontes do imaginário jurídico, o senhor identifica três campos de interação entre Direito e Literatura: o Direito da Literatura, o Direito como Literatura e o Direito na Literatura. O quanto há de interação e o quanto há de confronto entre o Direito e a Literatura?

Parlons d'abord des interactions. Comme déjà dit, elles sont nombreuses, ouvrant autant de champs, ou d'axes, dans le vaste domaine « droit et littérature ». Aux trois axes classiques que vous évoquez, j’en ajoute maintenant deux autres. D’une part, le « droit par la littérature » - par quoi je vise les textes littéraires écrits par des juristes ou des politiques en vue de faire avancer une «cause». On peut citer les pamphlets littéraires de Voltaire en vue de dénoncer les abus de l'ancien régime: arbitraire des lettres de cachet et intolérance religieuse dans les affaires Calas, et du Chevalier de la Barre. On pense aussi aux Lettres persanes de Montesquieu, aux nombreux textes de Victor Hugo luttant pour l'abolition de la peine de mort (Le dernier jour d'un condamné, Claude Gueux). J'ai moi-même écrit, si je peux me permettre, une Antigone voilée pour discuter la question de la légitimité du port du voile islamique dans l'espace public.

D'autre part, il y a aussi «la littérature comme droit » - des cas, exceptionnels sans doute, dans lesquels le corpus littéraire est utilisé comme source de droit en vue de résoudre des litiges. J'ai rencontré un exemple pareil à l'Université MacGill au Québec où est organisé, chaque année, un Moot court, procès fictif présenté à un groupe d'étudiants de droit et de lettres, avec pour consigne de discuter l'affaire à l'aide exclusive du corpus représenté par les pièces de Shakespeare. Aussi étonnant que cela paraisse, la formule fonctionne et, d'année en année, se constitue une «jurisprudence shakespearienne » qui, à son tour, vient enrichir le matériau disponible pour les futurs procès.

J'en viens maintenant aux confrontations entre droit et littérature . Elles aussi sont innombrables, depuis Les Lois et La République de Platon où dramaturges et poètes se font durement éconduire par les légistes au motif que leur art n'est pas sérieux et potentiellement subversif. Les deux variétés les plus connues d'opposition entre ces deux imaginaires rivaux (le roman politique officiel et la fiction littéraire) sont la censure et l'instrumentalisation ou l'embrigadement. L'artiste et le comédien sont souvent politiquement suspects au regard d'un pouvoir jaloux de ses 
prérogatives et méfiant à l'égard du bouffon qui lui rappelle qu'il est nu. Qu'il suffise d'évoquer le fait que, leur vie durant, des comédiens comme Molière et Shakespeare ont du composer avec la censure et un statut de paria qui, à tout moment, pouvait les rejeter au ban de la société. L'instrumentalisation ne représente pas un sort plus enviable pour l'œuvre littéraire - je pense à ces régimes politiques qui entendent mettre au pas et à leur service la production littéraire et artistique de leur temps. On peut évoquer par exemple la situation du théâtre à l'époque de la révolution française, sous Robespierre particulièrement. Il s'agissait de produire des œuvres patriotiques exaltant l'homme nouveau issu de la révolution, pétri de «vertu républicaine ». Inutile de dire que la qualité des œuvres ainsi portées au pinacle était pour le moins médiocre ; Sade en livre un pastiche éminnement subversif dans son Français, encore un effort si vous voulez être républicains !, opuscule inséré dans sa Philosophie dans le boudoir.

Dieter Axt - Bien que les États-Unis aient une production théorique assez vaste dans ce domaine, l'étude du droit et de la littérature se répand mondialement dans les dernières années. De quelle façon évaluez-vous le développement de cette étude?

Le bilan, sous réserve d'un meilleur inventaire, est mitigé. On trouve maintenant en Grande-Bretagne, aux Pays-Bas, en Italie, en Espagne, et aussi dans le monde hispanophone et lusophone des productions de qualité dans le domaine droit et littérature. En France, une revue intitulée «droit et littérature » vient de voir le jour chez l'éditeur Lextenso. Le domaine est sorti de sa marginalité : des colloques universitaires sont organisés, même sous l'égide de la Cour de cassation; des cycles de formation à destination des magistrats sont régulièrement mis en place dans le cadre de l' Institut des hautes études sur la justice (Paris); des sociétés savantes voient le jour, tenant réunions régulières. En revanche, trop rares sont les cours universitaires proposés aux étudiants dans ce domaine, l'orientation de leur formation restant marquée soit par le positivisme (poussant à l'accumulation de matières strictement juridiques), soit par l'utilitarisme (complétant l'offre de formation par des enseignements d'inspiration managériale). Personnellement j'ai la chance de dispenser un cours de 30 heures de droit et littérature, cours s'adressant 
à la fois à des étudiants de droit et de lettres, ce qui, de surcroît, permet d'utiles échanges entre ces deux groupes d'étudiants.

Je complète ma réponse en ajoutant cependant un développement significatif de l'intérêt pour des approches complémentaires : essentiellement droit et cinéma, mais aussi droit et musique, droit et art. Je me réjouis, bien entendu, de cet engouement nouveau, en rapport avec la place dominante de l'image et de la musique dans la culture contemporaine - moyennant cependant deux conditions. La première consiste à mettre en garde contre les facilités d'une approche «culturaliste» un peu vague, rapprochant le droit, sans beaucoup de rigueur, de tout et n'importe quoi. Ma deuxième remarque s’inscrit dans la même perspective: dans ces rapprochements nouveaux, il s'agit de prendre très au sérieux le langage de l'image et les techniques d'écriture musicale, exactement comme je m'efforce de prendre très au sérieux les spécificités de la pratique de l'écriture littéraire ou de la performance théâtrale. Ici aussi il faudra s’imposer l'ascèse d'un véritable décentrement et la discipline d'un authentique apprentissage de ces techniques spécifiques.

Dieter Axt - Au Brésil, la relation entre le droit et la littérature est un jeune domaine de recherche. L'émission télévisée hebdomadaire Direito \& Literatura, qui existe depuis six ans, a pour but de débattre des questions relatives à la pensée juridique par le biais de la perspective littéraire. De quelle façon la littérature peut-elle aider la recherche de réponses pour des questions dans le domaine du droit, de la justice et du pouvoir ? Quelle est l'importance de l'étude trandisciplinaire pour le droit?

Je ne connais pas cette émission; je serais très curieux de voir comment elle est menée et de quoi on y parle. De quelle façon la littérature fournit-elle des réponses? Je dirais: toujours de manière indirecte, oblique, imprévue. On ne cherche évidemment pas dans la littérature des réponses relatives à la technique juridique - sauf exception cependant. Balzac, qui avait travaillé dans une étude de notaire au lendemain de l'édiction du Code Napoléon (1804), traite, dans les nombreux romans qui composent sa Comédie humaine, d'aspects parfois extrêmement techniques du droit des contrats, de la faillite, du droit des personnes ou encore du droit successoral. Son œuvre est comme l'envers littéraire du Code civil : une exploration des coulisses du Code et une lumière crue jetée 
sur l'écart qui se creuse entre la lettre de la loi (ou l'intention du législateur) et ce que les acteurs en font réellement. Une plongée dans les noirs tréfonds de l'âme humaine, et une expérience inédite de passions qu'on peut qualifier de « juridiques », comme par exemple le fait de gagner un titre juridique, indépendamment de l'intérêt économique de ce titre (exemple d'une sorte de «fétichisme » juridique ; la folie procédurière plaider pour plaider - en est un autre exemple). J'observe aussi que W. Shakespeare est souvent cité, à la Cour suprême des Etats-Unis, à l'appui de tel ou tel argument technique. Mais ces cas sont plutôt rares, et, dans l'immense majorité des situations, la littérature nous éclaire sur l'anthropologie juridique, ou la sociologie politique, qui concernent non les détails de la solution juridique elle-même, mais plutôt son contexte humain, social - voire ses fondements éthiques et philosophiques. Bien entendu, il ne faut pas non plus, à l'inverse, diluer excessivement le champ de «droit et littérature»; c'est une des difficultés principales de l'exercice : trouver le juste milieu entre un usage purement technique de la littérature (illustrant telle discussion juridique de détail), et un usage complètement dilué de la fiction, mobilisée pour illustrer sans rigueur tel ou tel aspect mal identifié de l'ordre juridique. Il faut reconnaître au système juridique (à décliner au pluriel, du reste) une autonomie au moins relative et une nécessaire technicité (son efficacité sociale, sa capacité de transformation du réel passe par cette autonomisation technique souvent mal comprise et trop facilement décriée). La littérature ou l'art en général n'ont pas à se substituer à ce geste d'autonomisation ; en revanche, il leur appartient de recontextualiser celle-ci, et de la discuter, le cas échéant.

Un troisième aspect de la question porte sur la transdisciplinarité. Dans mes travaux d'épistémologie je distingue à cet égard trois manières de pratiquer le rapprochement de disciplines diverses: la pluri (ou multi)-disciplinarité, l’interdisciplinarité, et la transdisciplinarité. A vrai dire, je suis plutôt à sceptique à l'égard de la multi-disciplinarité (qui, en se contentant de juxtaposer des discours scientifiques différents, échoue à les articuler; on est alors en présence d'une sorte de «babel » scientifique, ou dialogue de sourds), ainsi que de la transdisciplinarité (qui, en prétendant surmonter les barrières disciplinaires, croit produire une science nouvelle; souvent cependant ce sera au détriment des 
concepts et méthodes propres aux sciences en présence). Sauf rares moments d'inspiration transdisciplinaire (avec, véritablement, émergence d'une discipline nouvelle), je préfère le patient dialogue interdisciplinaire, que j'assimile à une pratique traductive - vous savez que je considère la traduction comme le paradigme dont notre monde éclaté et pluriel a besoin aujourd'hui, dans de nombreux domaines ; cf mon Traduire. Défense et illustration du multilinguisme (paru chez Fayard en 2009). J'ajoute encore que, en toute rigueur, entre droit et littérature, il ne s'agit que marginalement de confrontation de disciplines (ce n'est le cas que lorsqu'on confronte sciences juridiques et sciences littéraires). Comme dit plus haut, il s'agit plutôt de la confrontation de deux pratiques et de deux imaginaires.

Dieter Axt - À votre avis, quels sont les classiques les plus importants de la littérature? Y a-t-il une façon de les identifier?

Bien entendu, je ne vais pas énumérer une liste de classiques que vous connaissez aussi bien que moi. Il serait intéressant, en revanche, de comparer rigoureusement les auteurs enseignés dans les systèmes scolaires à différentes époques. On aurait alors un aperçu de ce que chaque société considère comme son patrimoine littéraire académique (lié à l'institution scolaire et à ses choix pédagogiques). Votre question soulève par ailleurs une redoutable difficulté pour tous ceux qui travaillent dans le champ « droit et littérature » : c'est la question des limites du corpus. On me pose souvent cette question : quelles sont les œuvres littéraires pertinentes au regard de cette approche? Quand on a évoqué les tragiques grecs, la Bible, Balzac, Dickens, Dostoiëvski, Melville, Cervantes, Camus, Kafka, et quelques autres, dont quelques auteurs plus récents (Schlink, Dürenmatt, J.-M. Machado de Assis, R. Graciliano...), a-t-on fait le tour de la question ? Quid du polar par exemple? Et où faire passer la ligne de démarcation définissant l'œuvre littéraire entre un simple compte rendu d'une affaire criminelle par un chroniqueur judiciaire, le compte rendu de la même affaire par un homme de lettres comme Gide, ou Giono (pour l'affaire Dominici), ou encore Stendhal réécrivant de façon totalement originale un procès d'assises dans son fameux roman Le rouge et le noir ? A toutes ces questions, je répondrais simplement qu'est littéraire ce que nous considérons comme tel (je rappelle 
à cet égard que Kafka est mort en ayant très peu publié et en ne se considérant nullement comme un écrivain). Ainsi, il n’y a aucune limite a priori au champ d'investigation du domaine «droit et littérature ». La plaidoirie d'un avocat, le réquisitoire du ministère public, voire même l'aveu du coupable ou le témoignage d'un proche peuvent constituer « une page d'anthologie », plus riche qu'un roman creux et bavard. Tout dépend de l'écoute du public qui se saisit de ce texte et en fait un objet littéraire ou non. Votre question ravive également chez moi, un souci assez obsédant: découvrir des textes qui font sens aujourd'hui pour penser le droit et la justice, dans le contexte de la mondialisation (controversée) et du pluralisme qui l'accompagne. Qui sont les Camus d'aujourd'hui ? Répondre à cette question demande une attention soutenue, une curiosité tous azimuts et une bonne dose d'humilité.

Dieter Axt - Dans Jupiter, Hercule, Hermès : trois modèles du juge, vous avez mené une étude sur les archétypes de magistrats et d’interprètes de la loi ; le juge Hermes y est montré comme celui qui est adapté aux défis du Droit post-moderne. La tâche de l'application du droit est-elle éminemment herméneutique ? Comment peut-on extraire du sens des textes?

Plus largement encore: dès lors que nous sommes des êtres de langage, nous exprimant en paroles et en textes, je pense que c'est l'ensemble des sciences humaines et sociales, et pas seulement les sciences juridiques, qui relèvent de l'art du comprendre. Le paradigme herméneutique les caractérise, polarisé par la question, infiniment relancée, du sens, plutôt que le paradigme analytique, hélas dominant aujourd'hui, qui croit pouvoir raisonner en termes de vérité non contestée et de clôture logique. Donc, oui, bien entendu, appliquer la règle de droit relève des méthodes herméneutiques. Au sein de ce paradigme, on peut tantôt emprunter les ressources des disciplines argumentatives (Perelman, Alexy), tantôt celles de l'herméneutique au sens strict (Gadamer, Ricoeur, Dworkin), tantôt celle de la traduction, tantôt encore celles de la pragmatique narratologique. Je pense que ces approches sont des alliées, plutôt que des rivales ; elles contribuent toutes à éclairer, sous un aspect original, les mécanismes de la production du sens - opération risquée et jamais assurée de détenir «la » bonne réponse. J’ajoute que, lorsqu'on 
observe la production des cours de justice contemporaine, il faut noter une montée en puissance de la méthode de «balance des intérêts » (et du principe de proportionnalité qui l'accompagne), au détriment de l'herméneutique des textes proprement dits (notamment pour la raison que ces textes, souvent d'ordre constitutionnel, sont à ce point vagues qu'ils renvoient directement le juge à une prise en compte directe des valeurs et intérêts en cause dans l'affaire). Mais, même si la mise en œuvre du test de proportionnalité soulève des questions spécifiques, elle ne nous fait pas sortir pour autant du paradigme herméneutique : c'est toujours de construction risquée du sens qu'il s'agit.

Dieter Axt - Dans la littérature, nous avons des personnages comme Azdak dans Le Cercle de craie caucasien, Portia dans Le Marchand de Venise, ou encore Humpty Dumpty dans Les Aventures d'Alice au pays des merveilles, qui peuvent représenter des modèles de la relation entre l'interprète et le texte légal. Peut-on dire que la lecture d'un classique est aussi importante pour celui qui juge que la lecture des codes?

Vos exemples sont excellents. On pourrait encore ajouter le personnage de Busiris, l'expert en droit de la guerre que Hector, le chef des Troyens, convoque dans La guerre de Troie n'aura pas lieu de Jean Giraudoux. D'une minute à l'autre il s'avère capable de tirer du même texte ou du même comportement deux interprétations diamétralement opposées. Jouant à plein son rôle critique, la littérature multiplie les portraits de juristes-sophistes s'autorisant à lire les textes sous l'angle politique qui conforte leur intérêt, sinon leur caprice. Nommer, dire, interpréter, est l'acte politique par excellence, la première des performances sociales ; une façon efficace de maîtriser et posséder ce qui ainsi est nommé. Bien entendu, il est d'autres formes de nomination et d'interprétation qui s'avèrent respectueuses des personnes et des choses ; ce sont elles qui font les bons juges - ce que j'appelle les «juges traducteurs ». Il faut lire à cet égard les exemples jurisprudentiels réels discutés, dans cette perspective d'herméneutique respectueuse de l'altérité, par des auteurs comme M. Nussbaum (Poetic justice) ou J. Boyd White (Law as translation).

Bien entendu ces exemples littéraires et ces discussions philosophiques sont du plus grand profit pour les magistrats, chargés de 
« dire le droit » au quotidien, et qui sont confrontés, dans une assez grande solitude, à des situations sociales extrêmement complexes à l'égard desquelles les textes de lois ne sont que d'un secours relatif. C'est l'expérience que je retire de mes contacts fréquents avec les magistrats français suivant les formations «droit et littérature» organisées par L'Institut des hautes études sur la justice. Le juge américain Breyer n'hésitait pas à déclarer, lors de son audition devant le sénat américain en vue de sa nomination à la Cour suprême des Etats-Unis, que « la lecture de la littérature était une des occupations les plus utiles à l'exercice de sa profession de magistrat ». Outre l'expérience de l'humanité qu'offre la littérature, dont T. Todorov disait qu'elle était « laboratoire expérimental de l'humain ", la fiction accoutume le juriste à passer du « cas » singulier à la règle généralisable. C'est ce que Kant appelait le «jugement réfléchissant » (par opposition au jugement déductif classique, le jugement « déterminant ») ; et - ce n'est pas un hasard - Kant explique encore que ce type de saut du cas au critère, du singulier au général, ne trouve à s'appuyer que sur des « exemples » ou « récits inspirants ».

Dieter Axt - Vous exercez des activités dans le domaine du droit, de la philosophie et de la dramaturgie. De quelle façon ces trois domaines peuventils se combiner et comment interagissent-ils dans votre vie particulière?

Droit et philosophie relèvent de mes activités professionnelles, de mes enseignements et de mes recherches. L'écriture dramatique prolonge ces activités, sans coupure nette, mais avec changement de registre néanmoins. Il y va d'une pratique originale d'écriture, une expérience nouvelle pour moi, qui s'accompagne, car il s'agit toujours de théâtre, de collaboration étroite avec metteurs en scène, comédiens, parfois compositeur et chorégraphe. Ce type de collaboration, qui a pour effet de transformer l'œuvre écrite, de la porter à des niveaux de puissance insoupçonnés, s'avère extrêmement gratifiant (à la mesure aussi des risques pris et des angoisses ressenties). Ceci dit sans même parler du public «vivant» qui, à sa manière, fait le spectacle en assurant sa réception, chaque fois différente. Avec ce type d'écriture en première personne, je passe de l'autre côté du miroir. Cela m'apprend à lire autrement les textes littéraires, et particulièrement les pièces de théâtre 
qui sont des «performances » au moins autant que des textes. J'apprends par exemple les potentialités du langage des corps, et aussi la vertu du silence ( il y a des silences bien plus éloquents que de longues tirades c'est un enseignement utile pour un professeur !). Parfois il y a un rapport de complémentarité entre mes travaux scientifiques et mes pièces de théâtre. Ainsi, après avoir écrit mon Sade et la loi (Paris, Odile Jacob) j'avais le sentiment de ne pas avoir épuisé le sujet ; je ne pouvais pas, sans verser dans un moralisme désuet, discuter certaines thèses du « divin marquis ». En revanche, en mettant en scène un débat imaginaire (basé néanmoins sur des faits historiques avérés) avec Portalis, le principal auteur du Code civil et futur ministre des cultes de Napoléon, je pouvais traiter, sur le plan des passions réelles, des questions laissées en friche dans l'essai scientifique. De même, mon Antigone voilée, déjà évoquée, me permettait de traiter à bras le corps, et dans un langage chargé d'une vraie humanité, les passions opposées suscitées par le voile islamique - une étude scientifique de ces questions serait restée à la surface des choses.

Dieter Axt - Le passage de la pensée mythique à la pensée raisonnable a eu comme résultat la transposition de la métaphysique classique au jusnaturalisme moderne. Pourtant, la théorie du droit semble suivre comme une victime des fictions, dont le contrat social, la norme fondamentale ou la volonté générale. Que reste-t-il des structures mythiques dans la soi-disant rationalitéjuridique?

Vous avez raison de le souligner. La pensée rationnelle moderne n'a pas fait disparaître la pensée mythique, mais l'a refoulée profondément. Avec les inconvénients du refoulé, lorsqu'il n'est pas reconnu et assumé explicitement : des retours brutaux et non contrôlés. Je plaide donc pour une approche de la raison qui sache articuler les rôles de l'imaginaire et de la spéculation rationnelle. Des rôles complémentaires. A l'origine de nos découvertes scientifiques comme de nos institutions, opère toujours des constructions imaginaires. Comme l'écrit le philosophe Paul Ricoeur : «le symbole donne à penser »; le symbole, mais aussi le mythe et le récit. L'élaboration conceptuelle subséquente est essentielle, mais elle se nourrit toujours de cette orientation originelle. Nos constructions juridiques et politiques n'y font pas exception : elles reposent de plein pied sur ce que C. Castoriadis appelle « la constitution imaginaire» de la société. On parle 
aussi des peuples comme de «communautés narratives » fondées sur des « romans politiques» (Ch. Taylor). Loin d'être des enfantillages, ces mythes s'avèrent réellement fondateurs, tel le contrat social (la question n'est pas de le dissoudre, mais de le repenser à la hauteur des défis d'aujourd'hui, notamment en lui donnant une dimension planétaire et non plus seulement nationale, et en l'inscrivant sur une échelle transgénérationnelle plutôt que simplement contempopraine). Le droit tout entier repose sur des fictions performatives, petites ou grandes : la notion de «patrimoine commun de l'humanité », si nécessaire aujourd'hui, en est un exemple. La pensée managériale n'échappe en aucune façon à cet ancrage imaginaire : le mythe de la «main invisible », censée présider au marché et en réguler de façon optimale les échanges, en est un bon exemple. Le mythe d'une croissance possiblement infinie en est un autre.

Dieter Axt - Vous identifiez avec les personnages de Don Juan, de Robinson Crusoé et de Faust certains des rares mythes modernes de l'Occident. Pourquoi? De quelle façon agissent-ils sur notre imaginaire social ?

Je fais en effet la différence entre les classiques et les mythes. Les classiques sont des œuvres qu'on lit et qu'on relit. Les mythes, beaucoup plus rares, sont des histoires que l'on ne se contente pas de relire, mais qu'on réécrit périodiquement. Leurs ressources de sens sont si riches que chaque époque y trouve un matériau nécessaire pour se penser elle-même. G. Steiner écrit à cet égard que chaque époque, chaque culture a réécrit « son » Antigone. Faust, qui reprend lui-même, en amont, le mythe de Prométhée, et peut-être, plus en amont encore, l'histoire d'Adam, reste une métaphore de la condition humaine qui souffre pour s'être émancipée d'une contrainte. Certaines époques condamnent Faust, d'autres en font un héros (Goethe, par exemple, en fera le héros de l'Aufklärung). En revanche, le Faust médiéval qui pactise avec le diable est damné (chez Marlowe encore), tandis que le Docteur Faustus de Thomas Mann, contemporain du nazisme et symbole de la déchéance allemande, est à nouveau maudit.

Dieter Axt - Quelle est l'importance des tragédies grecques dans la création de l'imaginaire occidental ? Peut-on affirmer que les tragédies grecques contenaient la fondation de principes? 
Etant de culture classique et ayant été nourri, dès l'école, à la sève de la culture grecque, j’aurais tendance à répondre par l'affirmative à cette question. Mais je le fais sans dogmatisme, ni exclusivité. On trouve dans d'autres cultures et d'autres traditions des ressources aussi riches et diversifiées. Qu'il me suffise de citer par exemple la source biblique, d'inspiration sémite, avec les incroyables histoires contenues dans la Genèse, dont l'épisode de Babel ou celui du déluge. Pour revenir à la tragédie grecque, il faut lire ce qu'en dit G. Steiner qui va jusqu’à affirmer qu'elles forgent le vocabulaire et la syntaxe dans lesquelles se pensent les interdits fondateurs. Ainsi d'Edipe roi et de l'interdiction de l'inceste, ou d'Antigone et de l'interdit de la confusion entre les vivants et les morts (Créon est puni car, en maintenant le corps mort de Polynice en surface, et en emmurant Antigone, vivante, sous la terre, il chamboule l'ordre des choses et provoque la confusion interdite). Une pièce comme les Euménides d'Eschyle, quant à elle, contribue à fonder imaginairement le passage de la vengeance privée à la justice publique (la justice du tiers) - ce qui représente, sans conteste, l'indice le moins contestable du passage au droit. Mais les lectures des tragédies, comme leurs traductions, n'arrêtent pas d'évoluer et de s'enrichir. Ainsi N. Loreaux fait remarquer, avec raison, qu'en donnant très souvent la parole et le rôle central aux femmes, dans une culture très machiste, la tragédie représente la face cachée et refoulée de la cité, l' « autre » de la politique - l'inverse de la gloire et de l'épopée : le souvenir des morts et le rappel de la division sociale.

Dieter Axt - D’après vous, quelles sont les principales questions éticojuridiques que pose la globalisation et de quelle façon la littérature de nos jours se positionne-t-elle par rapport à ces questions?

La mondialisation pose une foule de questions éthico-juridiques, et une littérature immense leur est consacrée. Je me contente d'en citer deux. La première est relative à une exigence virulente de solidarité et de justice sociale, à l'heure où certains écarts se creusent au lieu de se combler, en même temps que progressivement, dans l'imaginaire collectif, les frontières de la communauté éthiquement pertinente (celle au sein de laquelle une exigence de solidarité peut légitimement se faire entendre) tendent à s'élargir aux limites de la planète entière. Non sans, bien 
entendu, que s'exacerbe aussi, en contrepoint, un sentiment d'insécurité et un repli égoïste à l'abri de frontières plus restreintes. La deuxième question est relative aux référents culturels et à une nouvelle définition de l'universel : la mondialisation se traduira-t-elle par une uniformisation réductrice et stérilisante, façon macdonaldisation du monde, ou, au contraire, par un enfermement identitaire dans des communautés intolérantes ? Les sociétés occidentales, bercées depuis des décennies, dans un individualisme exacerbé et plutôt hédoniste, manquent de ressources imaginaires et morales pour affronter ces questions, d'autant plus aiguës qu'on commence aussi à s'apercevoir des limites du modèle croissanciel qui soutient le discours politique depuis des décennies. S’il est vrai, à en croire J.-F. Lyotard, que la post-modernité a emporté la « fin des grands récits ", au premier rang desquels le discours d'émancipation marxiste et le discours de solidarité chrétienne, il ne nous reste qu'une quantité de « petits récits » dont on n'aperçoit pas encore lequel serait de nature à soutenir le projet mobilisateur d'une mondialisation solidaire et créative. Un chantier immense s'ouvre ici.

Dieter Axt - Est-ce qu'il y a un élément ou aspect que nous n'avons pas inclus dans nos questions dont vous aimeriez parler?

Tout au long de cet entretien, tant les questions que les réponses ont entretenu l'image d'un usage positif, fondateur, instituant, et émancipateur $\mathrm{du}$ récit. Cela correspond à ma conviction profonde. Je ne voudrais cependant pas, avant de conclure, passer sous silence les mauvais usages possibles du récit. Je songe au storytelling en particulier, cet art de « raconter des histoires et de formater les esprits », comme l'écrit Chr. Salmon. Omniprésent dans nos sociétés contemporaines, il exerce des effets normatifs puissants aux antipodes du projet solidaire et émancipateur : qu'il s'agisse de vendre un objet dans le marketing, un homme politique dans la propagande, une idéologie sectaire dans le discours des télévangélistes, une vision géopolitique dans le discours de politique étrangère (« la croisade contre le mal » de G. Bush), pas un domaine de la vie sociale ne lui échappe désormais. Il faudrait étudier de près, dans une perspective critique, les procédés narratifs utilisés par ces fictions de pacotille et les effets sociaux attendus de celles-ci. Il est 
significatif par exemple qu'on puisse faire coïncider avec beaucoup de précision, les cartes américaines des Etats ayant massivement voté en faveur de D. Trump et celles des taux d'écoute les plus élevés des séries télévisées les plus basiques. Ainsi donc, notre intérêt pour le récit ne doit jamais endormir notre vigilance critique ; précisément parce que leur rôle est décisif, les récits sont susceptibles du meilleur et du pire.

Sophocle nous avertit : à l'origine des cités, ce n'est pas la raison qui opère mais des «astunomous orgas ») (dixit le chœur dans le premier stasimon de l'Antigone) : expression qu'on peut traduire par «passions instituantes ». Des « passions » collectives et politiques : la peur, dans le pire des cas ; la solidarité et la liberté dans le meilleur des cas. Espérons que nous sachions prêter l'oreille au chant du poète qui, «veilleur insomniaque », se tient aux portes de la cité et se fait le porte parole des sans-voix, ceux qui, précisément, réclament « droit de cité ». 